\title{
V7: Bedarf an hochschulisch qualifizierter Pflege - Präsentation der Ergebnisse einer qualitativen Studie
}

\author{
Anke Simon · Bettina Flaiz $\cdot$ Melanie Elze
}

Online publiziert: 30. Oktober 2013

(C) Springer-Verlag Wien 2013

Hintergrund: Begründet in der zunehmenden Komplexität der Versorgung stellt die höhere Qualifikation der Pflegenden einen Lösungsansatz dar. Erste Absolventen grundständiger Pflegestudiengänge sind zu verzeichnen. Aussagekräftige Studienergebnisse aus der Evaluationsforschung über die neuen, innovativen Lehr- bzw. Studienprogramme fehlen jedoch bisher. Das Forschungsprogramm des vom BMBF und der EU geförderten Projekts OPEN - OPen Education in Nursing - wird vorgestellt. Erste Ergebnisse der Pilotstudie zum Bedarf hochschulisch qualifizierter Pflegekräfte aus Sicht der Praxis werden präsentiert.

Methodik: In einem exploratorischen, qualitativen Forschungsansatz wurden Tiefeninterviews, mit Hilfe eines strukturierten Leitfadens durchgeführt. Auf der Basis des selektiven Sampling ( $n=12)$ wurden entsprechend definierter Einschlusskriterien Experten über vier Zielgruppen ausgewählt (Leitung von Bildungszentren/Pflegefachschulen; Pflegedirektion/oberes Pflegemanagement; Entscheidungsträger in Fachverbänden der Pflege; Geschäftsführung KH/ Krankenhausgesellschaft). Die Datenauswertung der transkribierten Interviews folgt der qualitativen Inhaltsanalyse nach Mayring.
Ergebnisse: Die Erhebungsergebnisse zeigten einen hohen generellen Bedarf an akademisch ausgebildeten Pflegekräften aus Sicht der Praxis. Neben den Beweggründen und Aufgabenfeldern konnten weitere aussagekräftige Ergebnisse zur Akademisierungsquote, Kompetenzzielen und Rahmenbedingungen eines optimalen Studienprogramms, aber auch Hürden und Barrieren für die hochschulische Qualifikation von Pflegekräften identifiziert werden. Die Studienergebnisse werden mit aktuellen Publikationen aus dem In- und Ausland reflektiert und diskutiert. U. a. verdeutlichen die Ergebnisse die Nähe zum Konzept der Erweiterten Pflegepraxis (ANP) und der damit verbundenen Forderung nach patientennahen Abschlüssen. Bemerkenswert ist ebenso die Experteneinschätzung bezüglich der Quote an hochschulisch Qualifizierten, die deutlich höher ist als die jüngste Empfehlung des Wissenschaftsrates. Allerdings wird ebenso deutlich, dass die Restrukturierungsmaßnahmen und die Gestaltung des Qualifikationsmix in der Praxis den Akademisierungsbestrebungen auf Hochschulseite deutlich hinterherhinken.
B. Flaiz $(\bowtie) \cdot$ A. Simon $\cdot$ M. Elze

Duale Hochschule Baden-Württemberg, Stuttgart, Deutschland

E-Mail: Flaiz@dhbw-stuttgart.de 